\title{
Qualité sanitaire du lait cru \\ tout au long de la filière \\ dans le district de Mbarara et \\ la ville de Kampala en Ouganda
}

\author{
N. Grillet 1,2 P. Grimaud 1,2* G. Loiseau ${ }^{3}$ \\ M. Wesuta ${ }^{4}$ B. Faye ${ }^{2}$
}

Mots-clés

Lait - Qualité - Hygiène des

aliments - Contamination biologique -

Préservation - Ouganda.

\begin{abstract}
Résumé
La qualité sanitaire du lait cru est une problématique importante en Ouganda, tant pour des raisons socio-économiques que sanitaires. L'étude conduite sur la filière informelle du lait cru en Ouganda a mis en évidence deux principaux points critiques pour la qualité du lait: le manque d'hygiène du lieu de production au lieu de consommation, et l'absence de système efficace de conservation limitant le développement des bactéries pendant le transport vers Kampala. La charge microbienne a atteint en effet des niveaux très élevés, proche de $2 \times 10^{6}$ unités formant colonie par millilitre sur le lait de ferme dans le bassin laitier de Mbarara au sud-ouest du pays, que le transport vers la capitale Kampala a multiplié par un facteur d'environ 150. II existe, au sein de cette filière informelle, des unités artisanales dites de pasteurisation. Le lait qu'elles produisent est certes exempt de bactéries à sa sortie mais la conservation sur plusieurs jours de ce produit surchauffé pourrait rendre ce procédé plus dangereux que bénéfique. Ces constats doivent alerter tous les acteurs de la filière informelle sur la nécessité de mettre en œuvre une stratégie d'amélioration de la qualité du lait, à la fois par une modification des pratiques courantes pour qu'elles deviennent plus respectueuses de l'hygiène, et par une amélioration de la conservation du lait au moyen de technologies telles que la réfrigération, la pasteurisation à petite échelle ou encore l'utilisation du système lactoperoxydase. Cette étude a permis d'acquérir des bases techniques et scientifiques pour appuyer les actions visant à améliorer la qualité du lait cru en Ouganda. Quelle que soit la stratégie qui sera adoptée par les autorités, elle ne pourra être mise en œuvre que si elle implique l'ensemble des acteurs de la filière.
\end{abstract}

\section{INTRODUCTION}

En Ouganda, pays largement rural (80 p. 100 de la population active), l'élevage représente, après les céréales, le second poste dans la constitution du produit intérieur brut agricole ; il est par ailleurs majoritairement représenté (50 p. 100) par la production bovine laitière (1). Le secteur laitier présente dans ce pavs une

1. Cirad, UPR Systèmes d'élevage, université de Makerere, Kampala, Ouganda.

2. Cirad, UPR Systèmes d'élevage, Montpellier, F-34000 France.

3. Cirad, UPR Qualité des aliments tropicaux, Montpellier, F-34000 France.

4. Mbarara University of Science and Technology, Mbarara, Uganda.

* Auteur pour la correspondance

Cirad, UPR Systèmes d'élevage, TA 30/A, Campus international de Baillarguet, 4398 Montpellier Cedex 5, France.

Tél. : +25641342 120; fax : +25641342355

E-mail : patrice.grimaud@cirad.fr dynamique très forte, liée aux conditions générales de l'évolution de la demande en Afrique subsaharienne (15). Pour répondre à cette demande, la production laitière ougandaise n'a cessé d'augmenter depuis une dizaine d'années. On estime aujourd'hui que 900 millions de litres sont produits par an au niveau national, dont 70 p. 100 seraient commercialisés au travers d'une filière formelle (produits transformés) et d'une filière informelle (lait cru).

Toutefois, le secteur laitier connaît des problèmes de qualité liés essentiellement à la prédominance de la filière informelle. En effet, le lait cru (vendu exclusivement sur le marché informel) représente aujourd'hui 80 à 90 p. 100 du lait commercialisé en Ouganda. Ce phénomène est lié à plusieurs facteurs : (i) les consommateurs privilégient le lait cru, pour des raisons de goût et de prix (22) ; (ii) la filière formelle a beaucoup souffert du monopole de la Dairy Corporation, usine de transformation du lait gérée par l'Etat ; 
(iii) la filière informelle s'est aujourd'hui organisée et propose la plupart du temps aux éleveurs des conditions d'achat bien plus intéressantes que les usines du secteur formel (12).

Le cheptel ougandais est, de plus, fortement affecté par des zoonoses majeures telles que la tuberculose ou la brucellose (16) dont la transmission par la consommation de lait cru représente un facteur de risque important. Le problème de la qualité sanitaire du lait dans la filière informelle se pose donc pour assurer la sécurité des consommateurs ougandais, pour maintenir le niveau économique du secteur laitier et pour permettre aux producteurs de crédibiliser leur produit (18). L'objectif de la présente étude visait à identifier les principaux points critiques tout au long de la filière, dans le but de proposer une stratégie d'action pour l'améliorer.

\section{MATERIEL ET METHODES}

\section{Zone d'étude}

L'étude a été menée dans le district de Mbarara, principal bassin de production laitière en Ouganda, et dans la capitale, Kampala, où le lait est acheminé et consommé (figure 1). Elle s'est déroulée de mai à octobre 2004, soit pendant la saison sèche. Dans le district de Mbarara, trois zones agroécologiques sont classiquement décrites : les zones pastorale, agropastorale et agricole $(12,18)$ qui abritent trois types d'élevage: (i) les élevages traditionnels, où les producteurs élèvent des animaux de race locale, les Ankolés, en race pure, présentant généralement une faible productivité (environ 1 à $3 \mathrm{l} / \mathrm{j} /$ vache) ; (ii) les élevages intermédiaires, avec des troupeaux composés d'animaux de races Ankolé indigène, Frisonne-Holstein, introduite dans le pays il y a une quinzaine d'années, ou de leurs métis (environ 3 à $5 \mathrm{l} / \mathrm{j} /$ vache); (iii) les élevages modernes où les éleveurs possèdent un troupeau majoritairement de race Frisonne-Holstein, en race pure à productivité plus forte (environ 5 à 7 l/j/vache).

\section{Observation de la filière}

La première partie de l'étude a consisté en une analyse de l'organisation de la filière qui s'est faite en deux phases. Des discussions informelles avec les acteurs et des observations des déplacements de camions ont d'abord permis d'obtenir une première image de la filière et notamment des flux de distribution du lait cru. Par la suite, des entretiens ont été réalisés auprès des acteurs choisis pour le prélèvement des échantillons dans le but d'appréhender, d'une part, leur positionnement dans la filière et, d'autre part, leurs pratiques de gestion de l'hygiène. Ces informations ont permis de mieux comprendre les causes de dégradation de la qualité du lait au cours de son passage de la ferme au consommateur.

\section{Plan d'échantillonnage}

Les premières observations ont permis d'identifier les grandes étapes de la filière (figure 2) et ainsi de constituer la base du plan d'échantillonnage. Les unités de pasteurisation (septième niveau du plan d'échantillonnage) peuvent apparaître hors du sujet d'étude, focalisé sur la filière lait cru. Il est donc important de préciser que sont appelées « unités de pasteurisation » des structures récentes, entièrement imbriquées dans la filière informelle, et pratiquant un traitement thermique artisanal du lait $\left(75\right.$ à $80{ }^{\circ} \mathrm{C}$ pendant deux heures). Elles ont été incluses dans le but d'analyser leurs effets en matière de qualité.

Les critères de choix retenus pour réaliser le plan d'échantillonnage (tableau I) proviennent des observations de la première phase et des études diagnostic réalisées préalablement $(8,12)$. Ils ont servi essentiellement d'appui pour quantifier le nombre d'échantillons à prélever à chaque niveau de la filière.

Au niveau des fermes, le nombre d'échantillons par zone agroécologique a été corrélé à la production totale de chacune d'elle. La répartition a aussi tenu compte des trois types d'élevages identifiés, avec une prise de 4, 6 et 5 échantillons, respectivement dans les élevages traditionnels, intermédiaires et modernes. Les élevages traditionnels n'ont pu être considérés qu'en zone pastorale, car ils sont pratiquement absents dans les deux autres zones.

Dans la zone agricole, seul le niveau de la ferme a été représenté, car la grande majorité du lait collecté est destiné au secteur formel. Le marché du lait cru dans cette zone reste donc limité à un niveau très local et n'influence pas la qualité du lait allant à Kampala.

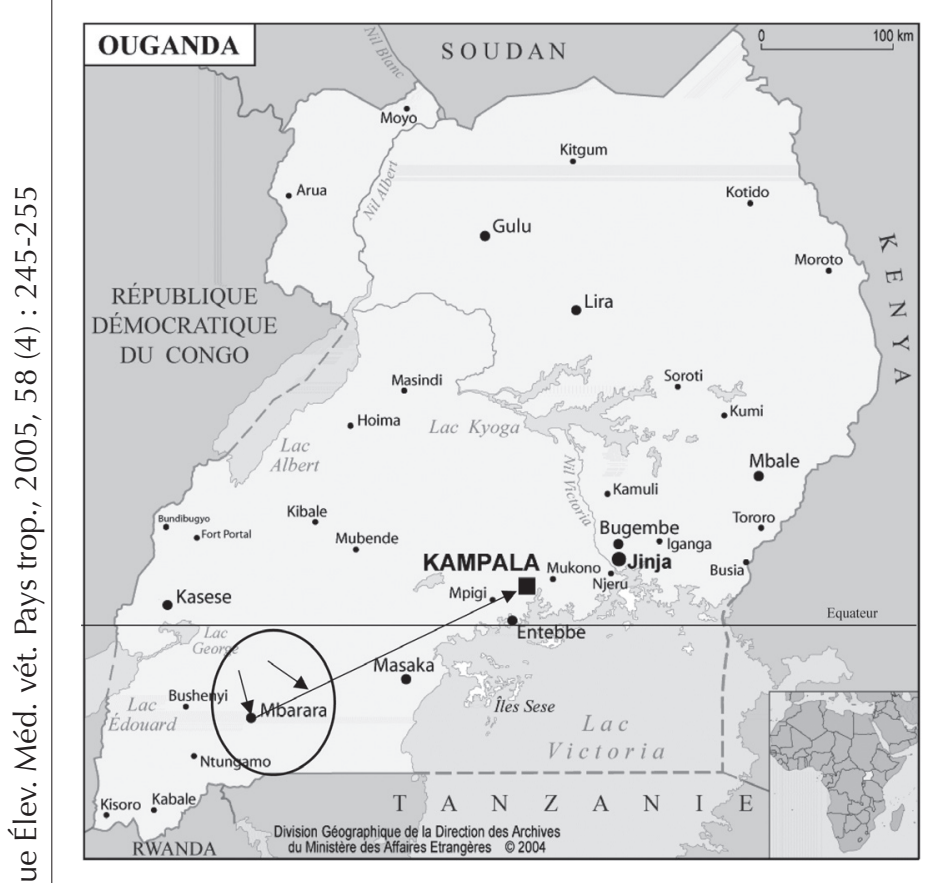

Figure 1 : carte générale de l'Ouganda et zones d'étude.

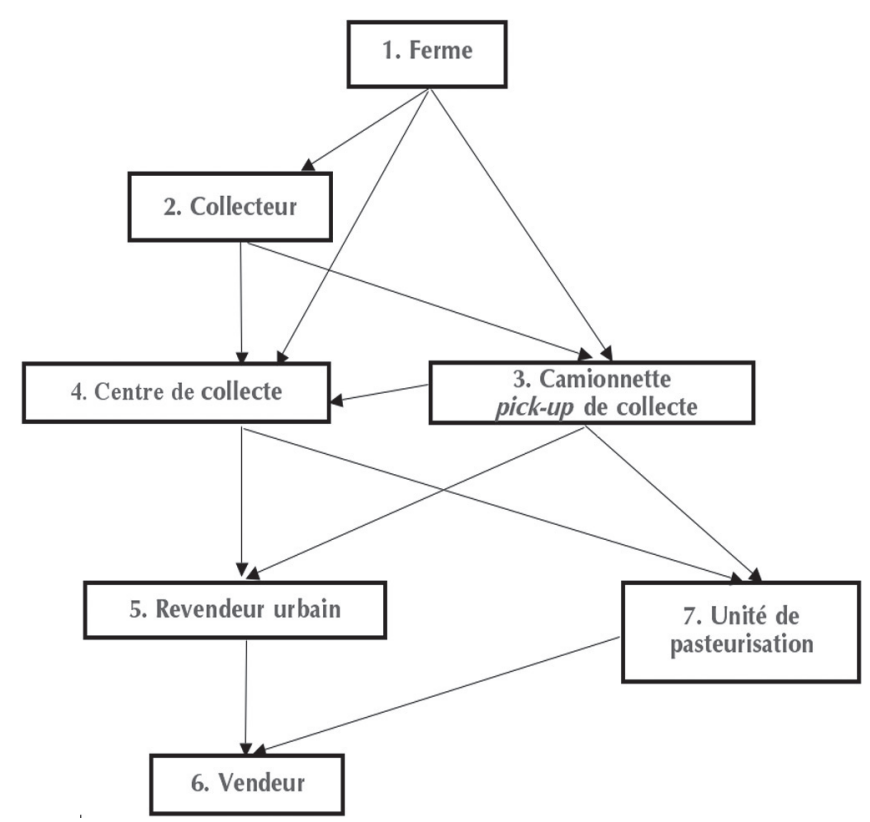

Figure 2 : organisation des sept niveaux d'échantillonnage dans la filière informelle. 


\section{Tableau I}

Répartition des échantillons en fonction des critères de choix à chaque niveau

\begin{tabular}{|c|c|c|c|c|c|}
\hline \multirow{2}{*}{$\begin{array}{l}\text { Niveau } \\
\text { Collecte }\end{array}$} & \multirow[t]{2}{*}{ Critères de choix } & \multicolumn{3}{|c|}{ Zone agroécologique } & \multirow{2}{*}{$\begin{array}{c}\text { Nb. d'échantillons } \\
(\mathrm{n}=\mathbf{8 8}) \\
41\end{array}$} \\
\hline & & Pastorale (19) & Agropastorale (20) & Agricole (2) & \\
\hline & Production de la zone & Forte (8) & Moyenne (5) & Faible (2) & \\
\hline Ferme & Type de ferme & $\begin{array}{l}\text { Traditionnelles (4) } \\
\text { Intermédiaires (3) } \\
\text { Modernes (1) }\end{array}$ & $\begin{array}{l}\text { Intermédiaires (2) } \\
\text { Modernes (3) }\end{array}$ & $\begin{array}{l}\text { Intermédiaires (1) } \\
\text { Modernes (1) }\end{array}$ & 15 \\
\hline Collecteur à vélo & Importance & Peu nombreux (2) & Nombreux (7) & Absents & 9 \\
\hline $\begin{array}{l}\text { Camionnette } \\
\text { pick-up de collecte }\end{array}$ & Propriétaire & $\begin{array}{l}\text { Grandes compagnies (2) } \\
\text { Indépendants }(1)\end{array}$ & $\begin{array}{l}\text { Grandes compagnies (2) } \\
\text { Indépendants }(2)\end{array}$ & $\begin{array}{l}\text { Destiné à } \\
\text { la filière formelle }\end{array}$ & 7 \\
\hline Centre de collecte & Refroidissement & Entrée (3) / sortie (3) & Entrée (2) / sortie (2) & $\begin{array}{l}\text { Destination } \\
\text { transformation }\end{array}$ & 10 \\
\hline Vente & & Mbarara (4) & \multicolumn{2}{|c|}{ Kampala (20) } & 47 \\
\hline Revendeur urbain & $\begin{array}{c}\text { Propriétaire } \\
\text { Refroidissement }\end{array}$ & $\begin{array}{l}\text { Indépendants (2) } \\
\text { Entrée (2) / sortie (2) }\end{array}$ & \multicolumn{2}{|c|}{$\begin{array}{c}\text { Grandes compagnies (7) } \\
\text { Indépendants (3) } \\
\text { Entrée (10) / sortie (10) }\end{array}$} & 24 \\
\hline Vendeur & Nombre & Absents & \multicolumn{2}{|c|}{ Très nombreux (14) } & 14 \\
\hline $\begin{array}{l}\text { Unité de } \\
\text { pasteurisation }\end{array}$ & $\begin{array}{l}\text { Traitement } \\
\text { thermique }\end{array}$ & Absentes & \multicolumn{2}{|c|}{$\begin{array}{c}\text { Entrée (3) / Sortie } 1 \text { (3) / } \\
\text { Sortie } 2 \text { (3) }\end{array}$} & 9 \\
\hline
\end{tabular}

Dans les centres de collecte et auprès des revendeurs urbains, deux prélèvements ont été effectués sur chaque lieu d'échantillonnage : un échantillon « entrée », prélevé à l'arrivée du lait sur place, et un échantillon "sortie », prélevé 10 à 24 h après le début du refroidissement. Ces prélèvements devaient permettre d'apprécier l'évolution de la qualité durant la phase de stockage au froid.

Sur le même principe, la qualité a été évaluée avant et après le traitement thermique dans les unités de pasteurisation. A ce niveau, un échantillon entrée et un échantillon sortie 1 ont donc été prélevés. A cela a été ajouté un échantillon sortie 2 prélevé plusieurs heures après la fin du traitement, afin de suivre la qualité du lait après le traitement thermique. Le nombre total d'échantillons s'est ainsi élevé à 88, répartis sur l'ensemble du district de Mbarara et de la ville de Kampala.

\section{Analyses de la qualité}

Les analyses pratiquées étaient celles généralement utilisées pour évaluer la qualité sanitaire du lait. Les protocoles répondaient aux normes internationales bien qu'ils aient parfois été adaptés localement par l'Uganda National Bureau of Standards (Unbs) ou régionalement par l'East African Community (EAC).

Neuf analyses ont ainsi été effectuées sur les 88 échantillons, dont cinq tests rapides :

- la mesure du $\mathrm{pH}$; lecture au pHmètre calibré par des solutions titrées de $\mathrm{pH} 4,7$ et 10 (30);

- la mesure de la densité ; lecture au lactodensimètre, accompagnée d'une mesure de température permettant de corriger la valeur lue sur le densimètre (24);

- le test à l'éthanol ; le mélange du lait à un volume équivalent d'éthanol à 70 p. 100 provoque une coagulation si le lait est anormalement contaminé (24) ;
- le test d'ébullition ; les laits anormaux (colostrum, laits mammiteux ou contaminés) coagulent au chauffage (24) ;

- le test à la résazurine ; $10 \mathrm{ml}$ de lait sont mélangés à $1 \mathrm{ml}$ d'une solution de résazurine standard, puis incubés 10 min à $37^{\circ} \mathrm{C}$. La coloration du mélange indique son niveau de contamination (sept niveaux) (30).

Les quatre analyses restantes ont été :

- le dénombrement de la flore aérobie mésophile totale sur gélose blanche ; dénombrement à plusieurs dilutions différentes après $48 \mathrm{~h}$ à $35^{\circ} \mathrm{C}$ [Mbarara University of Science and Technology (Must), Ouganda] ;

- l'énumération des coliformes totaux ; estimation selon la production de gaz en bouillon à la tryptose et au lauryl sulfate (trois tubes par dilution, trois dilutions incubées $48 \mathrm{~h}$ à $35^{\circ} \mathrm{C}$ ) et confirmation par production de gaz en bouillon lactosé bilié au vert brillant (48 h, $35^{\circ} \mathrm{C}$ ) (Must, Ouganda) ;

- l'énumération des coliformes fécaux ; isolement à partir des coliformes totaux et estimation selon la production de gaz en bouillon $\mathrm{EC}\left(48 \mathrm{~h}, 45^{\circ} \mathrm{C}\right)$ (Must, Ouganda);

- l'énumération d'Escherichia coli ; isolement à partir des coliformes fécaux et estimation selon le développement sur gélose à l'éosine et au bleu de méthylène $\left(24 \mathrm{~h}, 35^{\circ} \mathrm{C}\right)$, confirmation par test indole sur les colonies formées (Must, Ouganda).

La recherche de Mycobacterium sp. et de Listeria sp. a été effectuée en parallèle par le laboratoire de l'Unbs qui a ensuite communiqué ses résultats. Ces deux analyses ont été réalisées sur les échantillons de lait provenant des fermes et des revendeurs urbains, soit 41 échantillons représentatifs des premier et dernier niveaux de la filière, en vue de connaître l'importance de ces deux germes pathogènes majeurs. 


\section{RESULTATS}

\section{Qualité du lait à la production}

Plusieurs des analyses effectuées ont été considérées comme des indicateurs de la qualité globale du lait et de l'hygiène des pratiques (tests rapides, dénombrement de la flore totale et dénombrement des coliformes). Ceux-ci ont permis de constater des contaminations importantes au début de la filière (tableau II, figure 3), puisque, dès le niveau de production en ferme, la charge microbienne s'est élevée en moyenne à $1,84 \times 10^{6} \mathrm{ufc} / \mathrm{ml}^{1}$, puis a augmenté assez rapidement en passant d'un niveau à l'autre de la filière. Il est toutefois à noter que la dispersion des résultats autour de la moyenne (écarts moyens) est très importante, témoignant ainsi de la variabilité des pratiques d'hygiène d'un lieu de collecte à l'autre.

De même, les coliformes, témoins de l'hygiène des pratiques, étaient présents dès le niveau de la ferme (figures 4 et 5) à des valeurs plutôt élevées : respectivement $260 \mathrm{NPP} / \mathrm{ml}^{2}$ et $157 \mathrm{NPP} /$ $\mathrm{ml}$ pour les coliformes totaux et fécaux. A la différence de la flore totale, les coliformes se sont développés rapidement et ont atteint des taux importants avant même de quitter le district de Mbarara pour la vente. Néanmoins, ces constats étaient à relativiser car la variabilité des résultats pour les coliformes était à nouveau assez élevée. En effet, les écarts moyens à la ferme étaient respectivement de 335,73 et $206,31 \mathrm{NPP} / \mathrm{ml}$ pour les coliformes totaux et fécaux. L'énumération d'Escherichia coli a montré une évolution de la contamination qui suit de près les résultats obtenus pour les coliformes fécaux et qui n'a donc pas été détaillée ici.

Les résultats communiqués par l'Unbs pour la détection des Listeria sp. ont révélé 12 p. 100 de positifs. Sur ces cinq échantillons, trois provenaient d'élevages et deux de revendeurs urbains. Leur caractéristique commune a été qu'ils provenaient tous d'endroits où l'hygiène générale était plus satisfaisante que la moyenne, notamment, le matériel y était nettoyé à l'eau chaude et les personnes qui manipulaient se lavaient les mains. Les résultats de la détection de Mycobacterium sp. se sont quant à eux révélés tous négatifs.

\section{Qualité du lait et conditions de transport}

Les résultats d'analyses ont souligné l'importance de la dégradation du lait pendant le transport entre le district de Mbarara et la ville de Kampala. Le lait restait à température ambiante durant

\section{Tableau II}

Résultats moyens du dénombrement de la flore totale en début de filière

\begin{tabular}{lc} 
Niveau & $\begin{array}{c}\text { Moyenne flore totale } \\
\text { (écart moyen) } \\
\text { x 106 } \mathbf{~ u f c / m l}\end{array}$ \\
\hline Ferme & $1,84(3,22)$ \\
Collecteur & $1,22(1,21)$ \\
Camionnette pick-up de collecte & $171,39(285,32)$ \\
Centre de collecte entrée & $9,45(9,68)$ \\
Centre de collecte sortie & $6,37(5,40)$ \\
Centre de collecte moyenne & $7,77(7,55)$
\end{tabular}

${ }^{1}$ Unités formant colonie par millilitre

${ }^{2}$ Nombre le plus probable par millilitre

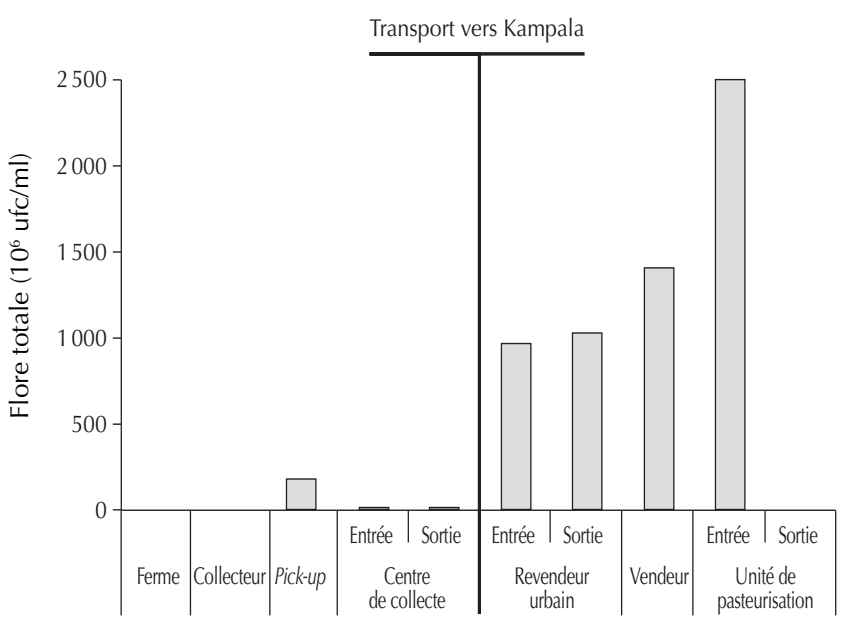

Figure 3 : résultats moyens du dénombrement de la flore totale par niveau.

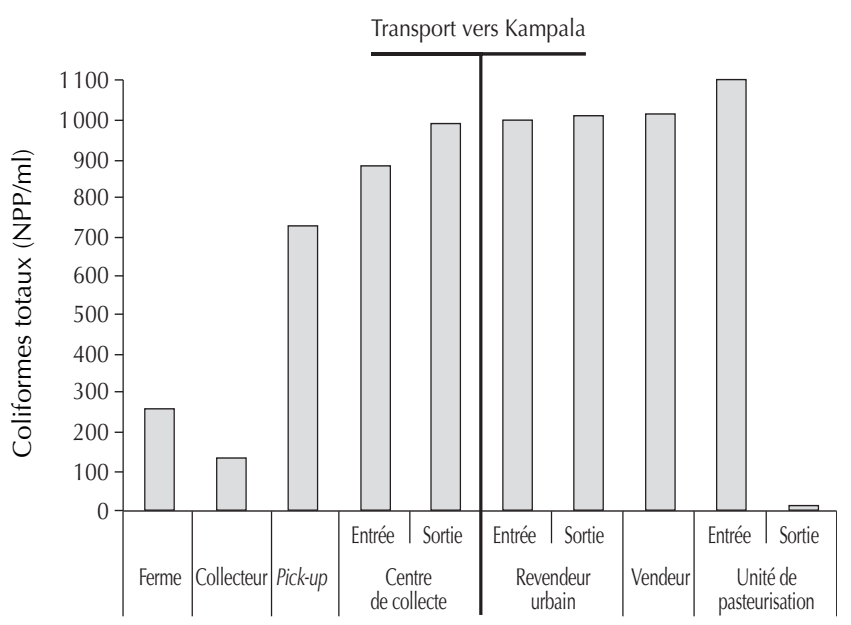

Figure 4 : résultats moyens du dénombrement des coliformes totaux par niveau (NPP = nombre le plus probable).

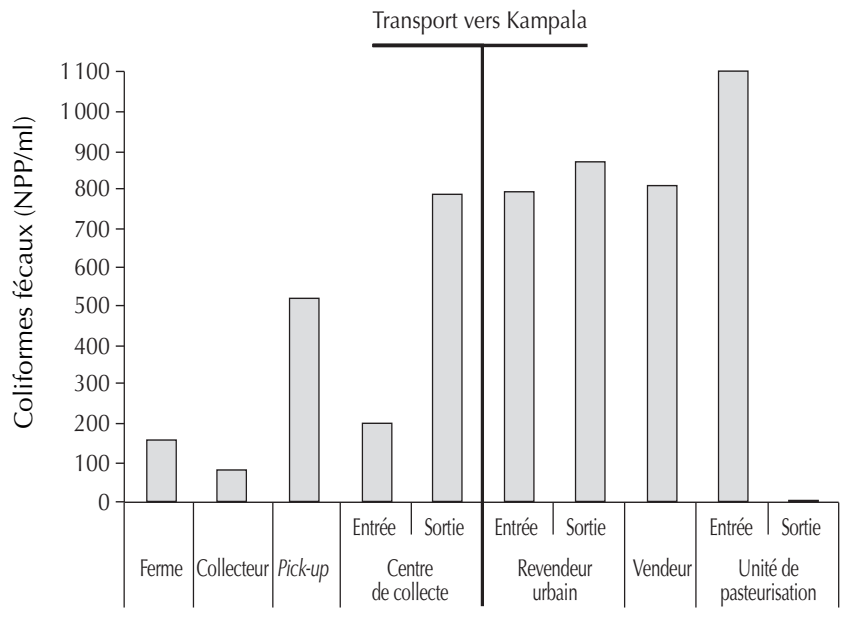

Figure 5 : résultats moyens du dénombrement des coliformes fécaux par niveau (NPP = nombre le plus probable). 
les 4 à 5 heures de transport en pleine journée, ce que Faye et Loiseau (17) définissent comme des conditions particulièrement favorables au développement rapide des bactéries. Or, le lait étant déjà fortement contaminé au départ de Mbarara $\left(6,37\right.$ x $10^{6} \mathrm{ufc} / \mathrm{ml}$ en moyenne à la sortie des centres de collecte), la charge microbienne connaît alors une croissance exponentielle (figure 3) multipliant par plus de 150 le nombre de micro-organismes par millilitre. Cette dégradation de la qualité globale a été confirmée par l'évolution des notes au test à la résazurine (figure 6) qui sont passées en dessous du niveau acceptable dès la sortie des revendeurs urbains, première étape du circuit de vente dans Kampala. En revanche, le transport n'a pas particulièrement favorisé le développement des coliformes puisque ceux-ci ont semblé effectuer leur phase de multiplication beaucoup plus rapidement que les autres groupes de bactéries (figures 4 et 5 ) et ont atteint quasiment le niveau maximum de détection permis par l'analyse (1 $100 \mathrm{NPP} / \mathrm{ml})$ lors de leur entrée au centre de collecte. Toutefois, les résultats de toutes les analyses ont montré une forte dispersion au niveau des vendeurs et des revendeurs urbains (tableau III), reflétant la variabilité des pratiques de gestion de la qualité sanitaire.

Par ailleurs, le lait transporté indirectement, c'est-à-dire refroidi en centre de collecte pendant 10 à $24 \mathrm{~h}$ avant d'être acheminé aux heures plus fraîches de la journée (à l'aube ou au crépuscule), a subi une altération moindre pendant le transport, en comparaison avec

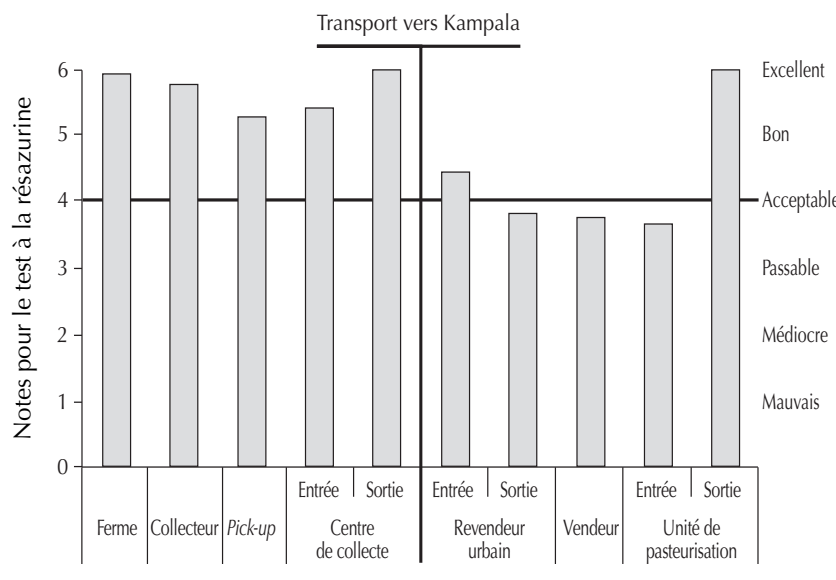

Figure 6 : moyenne des résultats du test à la résazurine par niveau (moyenne des notes sur 6). celui qui n'a pas été refroidi avant le départ (figure 7). L'ensemble des analyses montre en effet une stabilité des résultats dans le temps du lait refroidi sur place par rapport au lait transporté directement après la collecte. A titre d'exemple, la flore totale au moment de la vente aux particuliers (vendeur) a été pratiquement cinq fois plus nombreuse dans le lait n'ayant pas été refroidi avant le transport (4 $550 \times 10^{6}$ contre $932,43 \times 10^{6} \mathrm{ufc} / \mathrm{ml}$ pour le lait refroidi).

\section{Qualité du lait et pasteurisation}

L'ensemble des résultats d'analyse tendent à montrer que la pasteurisation a eu un effet stérilisateur sur le lait (figure 8). En effet, la flore bactérienne a été nulle pour la flore totale, $15 \mathrm{NPP} / \mathrm{ml}$ pour les coliformes totaux et une note de 6 à la résazurine à la sortie des unités de pasteurisation, et ce, malgré une qualité déplorable à l'entrée des unités (2 512,6 x $10^{6} \mathrm{ufc} / \mathrm{ml}$ pour la flore totale, 1100 $\mathrm{NPP} / \mathrm{ml}$ pour les coliformes totaux, note de 3,67 à la résazurine). Les échantillons sortie 2 ayant été prélevés peu de temps après la sortie des unités de pasteurisation, aucune différence significative n'a été notée entre ces échantillons et ceux prélevés immédiatement à la fin du procédé de pasteurisation (sortie 1). De plus, les écarts moyens en sortie des unités ont été très faibles (valeurs proches de 0 dans la plupart des cas), indiquant que les effets de la pasteurisation étaient constants d'une unité à l'autre.

Néanmoins deux résultats particuliers, inattendus après ce type de traitement thermique ont été notés : (i) dans certains cas, le $\mathrm{pH}$ augmentait après la pasteurisation pour retrouver un niveau normal (environ 6,4); (ii) les tests à l'éthanol étaient parfois positifs après pasteurisation (figure 9).

\section{DISCUSSION}

\section{Importance des pratiques d'hygiène}

Les résultats des analyses de la qualité globale (flore totale, tests rapides) indiquent tous une très mauvaise qualité du lait cru au regard des standards acceptés. A titre d'exemple, le seuil de 2 × $10^{6}$ ufc/ml, que l'EAC définit comme la limite maximale pour que le lait soit considéré comme acceptable (11), était largement dépassé avant même que le lait ne quittât la zone de production. Ces mêmes normes fixent à $10^{6} \mathrm{ufc} / \mathrm{ml}$ la valeur en dessous de laquelle le produit est jugé bon. La contamination du lait était déjà supérieure à cette limite au niveau de la ferme. Selon Faye et Loiseau (17), un animal sain dont la traite est effectuée dans de bonnes conditions d'hygiène produit normalement un lait peu contaminé contenant

\section{Tableau III}

Ecarts moyens (par rapport à la moyenne) des résultats des indicateurs principaux pour les revendeurs urbains (entrée, sortie) et les vendeurs

\begin{tabular}{lcrr} 
Analyse & \multicolumn{2}{c}{ Revendeurs urbains } & Vendeurs \\
& \multicolumn{1}{c}{ Entrée } & Sortie & \\
\cline { 2 - 3 } & & & \\
$\mathrm{pH}$ & $0,05(6,38)$ & $0,19(6,31)$ & $0,24(6,23)$ \\
Résazurine & $1,32(4,45)$ & $1,19(3,83)$ & $1,63(3,77)$ \\
Flore totale $\left(\times 10^{6} \mathrm{ufc} / \mathrm{ml}\right)$ & $1363,67(973,27)$ & $1310,25(1039,63)$ & $1674,42(1417,26)$ \\
Coliformes totaux $(\mathrm{NPP} * / \mathrm{ml})$ & $181,32(1000,27)$ & $161,49(1011,92)$ & $150,67(1018,38)$ \\
Coliformes fécaux $(\mathrm{NPP} / \mathrm{ml})$ & $391,54(792,36)$ & $347,13(868,58)$ & $405,16(807,38)$
\end{tabular}

* Nombre le plus probable 
une flore globale de $10^{3}$ à $10^{5} \mathrm{ufc} / \mathrm{ml}$. Ici, les charges microbiennes présentes dans les fermes ont été bien supérieures à ces valeurs indicatrices de bonnes pratiques d'hygiène.

Les coliformes totaux et fécaux quant à eux sont considérés comme des témoins de l'hygiène de traite en raison de leur origine fécale $(19,21)$. Or, ils ont été présents dans les échantillons provenant des fermes en quantités supérieures au seuil de $100 \mathrm{NPP} / \mathrm{ml}$ recommandé par l'Unbs (30). De telles valeurs témoignent de pratiques d'hygiène insuffisantes lors de la traite (17), confirmant ainsi les observations de Desvaux (10) qui rapporte, à titre d'exemple parmi une dizaine de pratiques à risque, que 71,4 p. 100 des éleveurs de la région de Mbarara ne se lavent jamais les mains avant la traite. Les pratiques de traite les plus courantes (tableau IV) peuvent ainsi être particulièrement mises en cause dans la contamination initiale du produit $(9,32)$. De plus, l'absence de contrôles de qualité à chaque étape de la filière et la mauvaise gestion de l'hygiène à tous les niveaux ont contribué à la dégradation rapide du lait cru pendant son acheminement final vers le consommateur.

La qualité du lait sortant des élevages présente un risque d'autant plus important que les troupeaux ougandais sont largement contaminés par la tuberculose, posant un problème sanitaire majeur dans le pays. En effet, en 2001, une étude portant sur 340 troupeaux du district de Mbarara a révélé un taux de prévalence troupeau pour la tuberculose de 74 p. 100 (16). Pourtant, les résultats des détections de Mycobacterium sp. effectuées sur les 41 échantillons de laits de fermes et de revendeurs urbains ont tous été négatifs, laissant supposer que la transmission du bacille par le lait était minime ou que le test effectué était peu fiable.

Les bactéries du genre Listeria présentent également un risque important pour la santé humaine et le lait cru en contenant est considéré comme impropre à la consommation au vu des normes
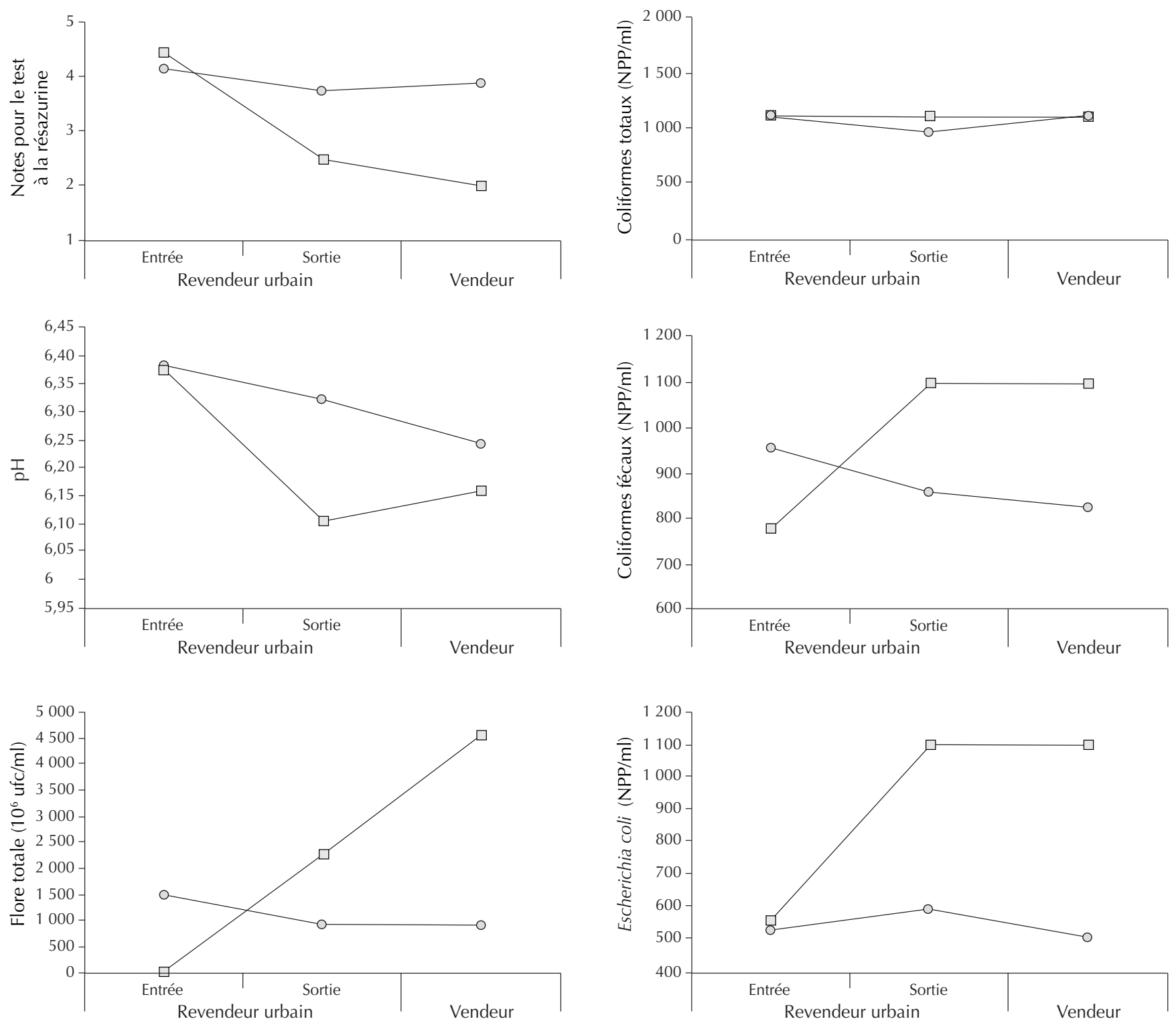

$\multimap-$ Refroidi $\quad-\square-$ Non refroidi

Figure 7 : effets du refroidissement avant transport sur six indicateurs de la qualité ; résultats chez les revendeurs urbains (entrée et sortie) et chez les vendeurs. NPP = nombre le plus probable. 

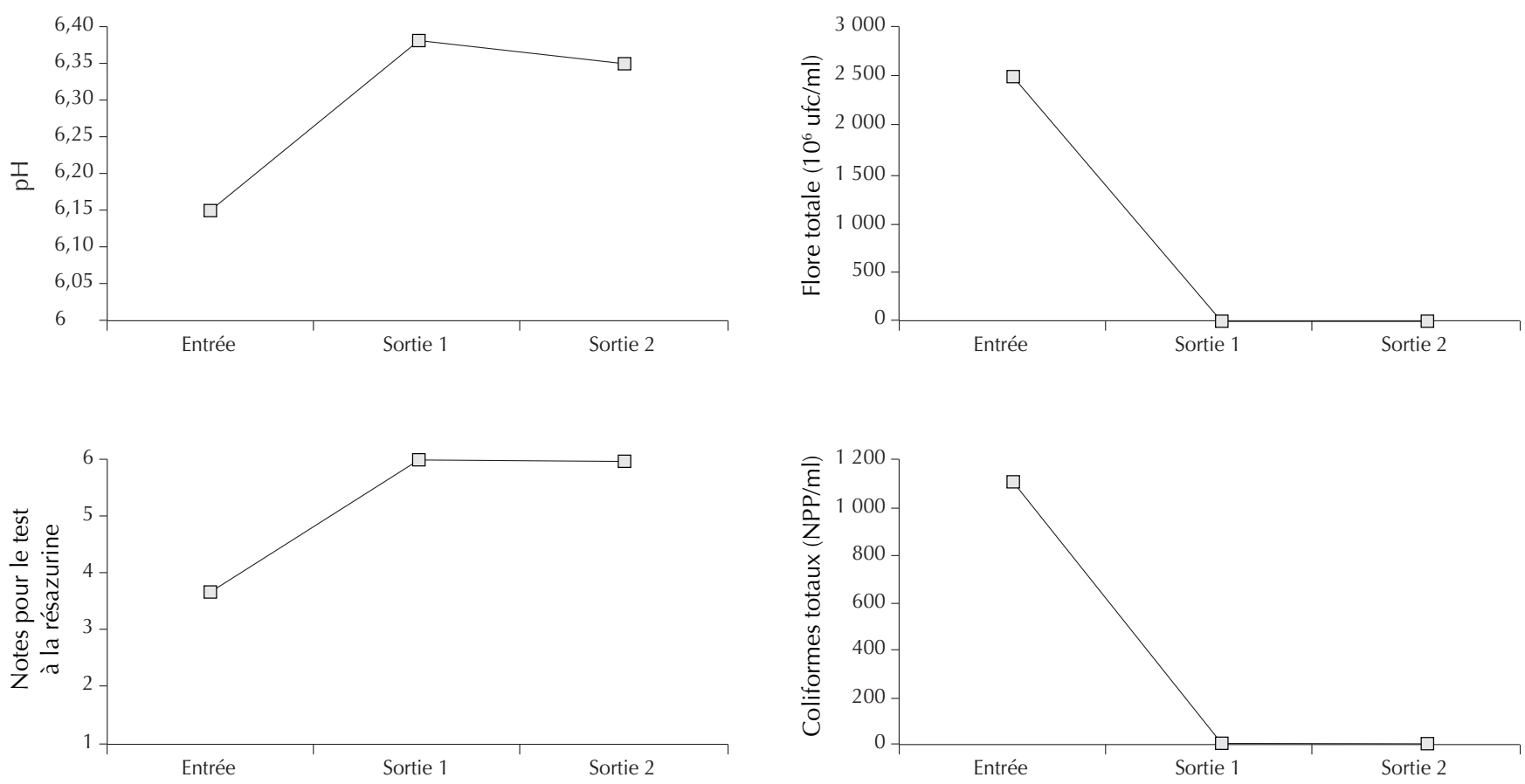

Figure 8 : effets du traitement thermique sur quatre indicateurs de la qualité globale; résultats des unités de pasteurisation (entrée, sortie 1 et sortie 2). NPP = nombre le plus probable.

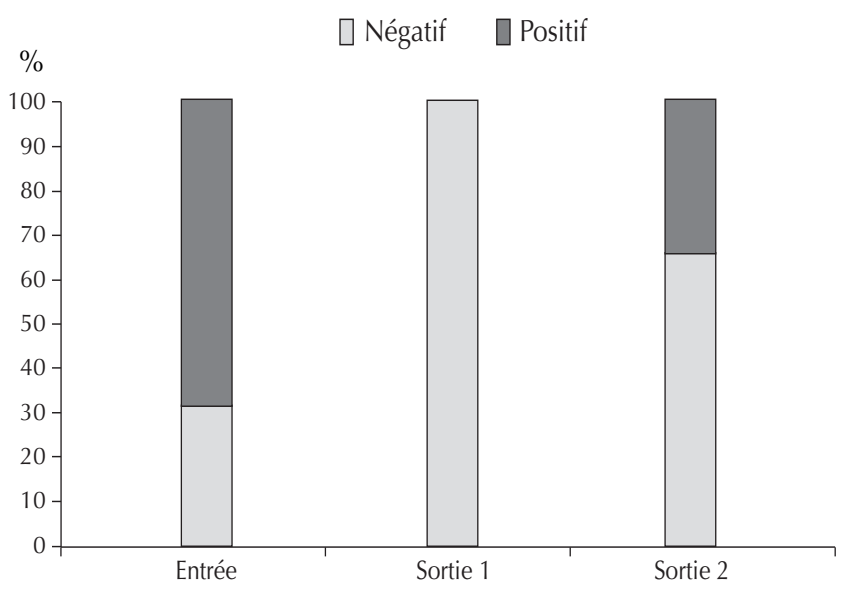

Figure 9: effet du traitement thermique sur les résultats du test à l'éthanol ; résultats des unités de pasteurisation (entrée, sortie 1 et sortie 2).

européennes (7). Les résultats proposés par l'Unbs concernant la présence des Listeria sp. ont montré que les échantillons positifs provenaient tous de lieux de collecte respectant mieux les règles d'hygiène que la moyenne. Ce phénomène s'explique par le fait que les Listeria sp. sont des bactéries opportunistes, s'accommodant mieux des milieux peu contaminés (29). Elles proviennent généralement de l'environnement (10) et peuvent donc témoigner d'un contact trop long du lait avec l'air, notamment durant la traite. De même l'eau utilisée pour le nettoyage des récipients est souvent contaminée (28, 32) et peut-être elle aussi un vecteur de bactéries telles que les Listeria sp. Ainsi, même aux endroits où l'hygiène était mieux maîtrisée, la qualité sanitaire du lait n'était pas encore optimale et le lait produit présentait un danger pour la santé des consommateurs.
La présente étude a ainsi permis de mettre en évidence l'insuffisance des pratiques d'hygiène, notamment au niveau de la production, par la publication de résultats scientifiques sur la qualité sanitaire du lait. Toutefois, il serait intéressant de poursuivre l'étude de la qualité globale (sanitaire et sensorielle) pour identifier exactement les pratiques à risque et ainsi mieux sensibiliser les acteurs de la filière. Ceci permettrait aussi de mettre en relation la composition de la flore microbienne du lait avec les pratiques d'élevage. Cette donnée pourrait être une information importante par la suite pour mieux contrôler la modification de la qualité organoleptique du lait lors des traitements thermiques (27) et ainsi favoriser leur acceptation par les consommateurs.

\section{Refroidissement et qualité}

La comparaison entre les laits refroidis avant transport et ceux amenés directement aux revendeurs urbains de la capitale a permis de mettre en avant l'importance de cette étape de refroidissement au niveau des centres de collecte. En effet, en refroidissant le lait peu de temps après la traite, on profite des qualités bactériostatiques du produit (17) et les bactéries sont bloquées en phase de latence où la croissance est quasiment nulle (33). Dans le cas du lait non refroidi, les bactéries terminent leur phase de latence pendant le transport et entament un développement important avant que le lait n'atteigne sa température de conservation. Cette étape, même si elle retarde le départ du lait collecté pour la capitale, permet ainsi de stabiliser le produit à son niveau de qualité de départ. Bien entendu, l'efficacité de cette technique repose entièrement sur l'amélioration des pratiques d'hygiène en amont des centres de collecte.

\section{Effets de la pasteurisation}

La pasteurisation en vrac a été proposée par la Dairy Development Authority (DDA) pour améliorer la qualité du lait par un traitement thermique final avant la vente. Ceci a été mis en œuvre par la création d'unités de pasteurisation artisanales. Les résultats concernant 


\section{Tableau IV}

Sources de contamination liées aux pratiques de traites *

\begin{tabular}{|c|c|c|}
\hline Agent de contamination & Sources potentielles & Dangers \\
\hline \multirow[t]{3}{*}{$\begin{array}{l}\text { Animal ; contaminations } \\
\text { endogènes }\end{array}$} & $\begin{array}{l}\text { Vache atteinte de mammite } \\
\text { non détectée }\end{array}$ & $\begin{array}{l}\text { Staphylococcus sp., E. coli, dégradation nutritionnelle, } \\
\text { destruction des protéines, réduction de la durée de conservation }\end{array}$ \\
\hline & $\begin{array}{l}\text { Vache porteuse saine } \\
d^{\prime} \text { une maladie }\end{array}$ & $\begin{array}{l}\text { Mycobacterium sp., Brucella sp., Staphylococcus aureus, } \\
\text { Streptococcus sp., Listeria sp., E. coli }\end{array}$ \\
\hline & $\begin{array}{l}\text { Non-respect du temps } \\
\text { d'attente après traitement }\end{array}$ & $\begin{array}{l}\text { Résidus chimiques, antibiotiques, anti-parasitaires, } \\
\text { inhibition de la lactofermentation }\end{array}$ \\
\hline \multirow[t]{2}{*}{$\begin{array}{l}\text { Animal ; } \\
\text { contaminations exogènes }\end{array}$} & $\begin{array}{l}\text { Pas de nettoyage de la mamelle } \\
\text { et des trayons, pas de séchage }\end{array}$ & $\begin{array}{l}\text { E. coli, Salmonella sp., Clostridium sp., coliformes et autres } \\
\text { bactéries fécales, bactéries psychrotrophes, bactéries lactiques }\end{array}$ \\
\hline & Queue non attachée & $\begin{array}{l}\text { Contamination par des particules de déjections et de terre, } \\
\text { coliformes et autres bactéries fécales, Salmonella sp., } \\
\text { bactéries psychrotrophes }\end{array}$ \\
\hline Personne à la traite & Pas de lavage des mains & $\begin{array}{l}\text { Staphylococcus sp., Streptococcus sp., E. coli, Salmonella sp., } \\
\text { Clostridium sp., bactéries fécales }\end{array}$ \\
\hline \multirow[t]{3}{*}{ Contenants } & $\begin{array}{l}\text { Mauvais nettoyage, } \\
\text { mauvais séchage }\end{array}$ & $\begin{array}{l}\text { Multiplication des bactéries se nourrissant des résidus, } \\
\text { contamination par l'eau, E. coli }\end{array}$ \\
\hline & $\begin{array}{l}\text { Transfert brutal } \\
\text { entre deux contenants }\end{array}$ & $\begin{array}{l}\text { Dégradation nutritionnelle, réduction de la durée } \\
\text { de conservation, rancissement }\end{array}$ \\
\hline & $\begin{array}{l}\text { Absence de filtration, } \\
\text { filtre mal nettoyé }\end{array}$ & $\begin{array}{l}\text { Contamination par des particules de déjections, de terre, } \\
\text { des insectes, des plantes... entraînement des souillures } \\
\text { retenues dans le filtre }\end{array}$ \\
\hline \multirow[t]{2}{*}{ Environnement } & Lieu de traite non nettoyé & Contaminations fécales, coliformes \\
\hline & Contact prolongé avec l'air & $\begin{array}{l}\text { Germes de l'environnement : Listeria sp., Pseudomonas sp., } \\
\text { bactéries psychrotrophes, levures et moisissures }\end{array}$ \\
\hline
\end{tabular}

* De Graaf et coll., 1997 ; Desvaux, 2001 ; Faye et Loiseau, 2002 ; Jouzier et Cohen-Maurel, 1986 ; Tourette et coll., 2002 ; Weber, 1985

ce traitement ont semblé effectivement indiquer une amélioration de la qualité globale par l'élimination des bactéries. Cependant, le chauffage excessif dans ces unités semble provoquer une altération physico-chimique du lait et une dénaturation des protéines (3) qui coaguleraient alors à l'éthanol. On peut aussi penser que cette altération provoque la destruction partielle ou totale des systèmes naturels de stabilisation et de protection du produit, comme le système lactoperoxydase par exemple (27). Ceci signifierait donc que le lait dit pasteurisé produit dans ces unités deviendrait un substrat vierge idéal pour une nouvelle colonisation par les bactéries environnantes, notamment pour les opportunistes telles que Listeria sp. (3).

Ainsi, même si l'effet bactéricide d'un tel traitement est efficace, plusieurs facteurs de risque persistent : (i) le traitement pratiqué est agressif, essentiellement à cause du temps de chauffage appliqué qui dépasse de beaucoup celui de trente minutes (temps maximal) défini officiellement pour la pasteurisation du lait (33) ; (ii) les conditions de vente du lait pasteurisé ne permettent pas d'assurer le maintien de la qualité obtenue $(2,26)$ : les emballages utilisés ne sont pas stérilisés, le matériel est nettoyé de façon rudimentaire et le lait pasteurisé n'est pas stocké au froid.

De plus, la modification des qualités organoleptiques du produit par des réactions physico-chimiques (de type Maillard par exemple) et par la destruction de la flore (27) est une des raisons pour laquelle les unités de pasteurisation artisanales ne connaissent pas le succès escompté par la DDA. En effet, les consommateurs de lait cru ne retrouvent pas dans ce nouveau produit le goût qu'ils recherchent. Leur difficulté à pénétrer le marché confronte les unités de pasteurisation artisanale à un problème économique majeur : pour être compétitives, elles se voient obligées de vendre au même prix que les vendeurs de lait cru alors qu'elles ont un coût de production supérieur.

\section{Propositions pour une amélioration de la qualité sanitaire du lait cru}

\section{Développement de la chaîne du froid}

Le refroidissement est considéré comme l'un des meilleurs moyens de préservation du lait par gestion du risque de développement bactérien $(13,17)$. Le lait est un produit sensible, il se détériore rapidement et nécessite donc un système de refroidissement rapide et une chaîne du froid efficace et complète tout au long de la filière, difficile à mettre en place dans des conditions tropicales $(26,31)$.

En Ouganda, les infrastructures sont encore insuffisantes malgré un développement significatif ces dernières années (centres de collecte). Le réseau routier ne permet pas un transport très rapide et il existe peu de disponibilités en matière d'équipement frigorifique (20). 
En outre, la mise en œuvre d'une chaîne du froid en Ouganda devrait être précédée d'une étude socio-économique pour comprendre et évaluer le coût et l'efficacité d'une telle mesure sur l'ensemble de la filière. Il est probable que les grandes compagnies réussiront à s'adapter facilement aux nouvelles exigences, alors que les acteurs indépendants, trop petits pour supporter le coût de la chaîne du froid, se verront obligés, à long terme, de se regrouper pour survivre ou disparaîtront.

Néanmoins, il semble que c'est vers l'établissement d'une chaîne du froid complète sur l'ensemble du secteur laitier que l'Etat ougandais devrait porter son effort. Ce type d'organisation coûteux et long à mettre en place peut se faire en complément de stratégies alternatives, telles que l'utilisation des traitements thermiques à petite échelle, ou l'introduction du système lactoperoxydase, déjà mis en œuvre dans certains pays de la zone est africaine (23).

\section{Utilisation des traitements thermiques}

Comme les résultats de l'étude le montrent, les conditions de pasteurisation et de vente des unités artisanales ne permettent ni de garantir la qualité sanitaire et sensorielle optimale pour le consommateur, ni d'assurer la viabilité économique de l'activité. Pour pallier ce phénomène, des minilaiteries ou micro-unités de transformation ont été installées à titre expérimental dans le district de Mbarara. Elles permettent de transformer à moindre coût de petites quantités de lait en maîtrisant les contraintes d'hygiène en posttraitement. Ces unités ont l'avantage de dynamiser efficacement le tissu économique au niveau local et d'améliorer la qualité générale par des techniques innovantes et par la contraction de la filière (4).

Le développement de telles unités réparties sur le territoire semble donc être une possibilité intéressante pour l'amélioration de la qualité globale du lait. Toutefois, leur installation reste possible sans investissements trop importants à la condition qu'elles ne traitent que de petites quantités à un niveau très local. Il est donc difficilement envisageable de considérer ces micro-unités comme une solution à l'échelle de la filière informelle globale.

\section{Utilisation du système lactoperoxydase (SLP)}

La lactoperoxydase est une enzyme qui permet de préserver à moindre coût la qualité initiale du lait pendant plusieurs heures à température ambiante. Elle représente à ce titre une solution alternative pour améliorer les conditions de transport du lait dans les zones difficiles à équiper en matériel de refroidissement $(5,13)$.

Il existe aujourd'hui un kit de SLP pour le traitement de bidons de 50 l. Ce kit présente le double avantage d'être utilisable sur de petites quantités et d'être présenté sous la forme de deux comprimés (l'un de thiocyanate, l'autre de peroxyde d'hydrogène), limitant ainsi les erreurs de dosage (6). L'utilisation du complexe lactoperoxydase est simple tout en nécessitant un minimum de formation auprès des acteurs. Cependant, elle reste aujourd'hui interdite par la FAO sur le lait destiné à l'export (14), notamment pour des raisons d'image du produit.

Le SLP pourrait être introduit en Ouganda au travers d'un programme expérimental dans la région de Mbarara, uniquement sur la filière lait cru, qui s'adresse exclusivement au marché national et n'est donc pas soumise à la restriction concernant le marché international. Accompagnée par une démarche de sensibilisation auprès des producteurs, des gérants de points de collectes et des scientifiques locaux, cette expérience pourrait ouvrir sur une stratégie plus large de gestion de la conservation du lait au niveau national.
Toutefois, la pertinence de l'utilisation du SLP en comparaison à la mise en place de la réfrigération systématique reste à démontrer dans le contexte ougandais.

\section{CONCLUSION}

La qualité sanitaire des aliments répond à plusieurs enjeux. D'une part, elle est une condition nécessaire pour assurer la santé des consommateurs, d'autre part, la question de la qualité est essentielle au sein d'une filière, car elle conditionne en grande partie l'évolution économique de celle-ci. Le défi est donc non seulement de garantir la sécurité des aliments, mais aussi d'assurer au secteur un bon développement économique dans le temps.

Les résultats de l'étude ont permis de montrer que le manque de qualité sanitaire du lait arrivant à la capitale était dû à deux catégories de facteurs : (i) les sources de contamination sont liées essentiellement au manque d'hygiène en amont de la filière, notamment au niveau de la ferme ; (ii) les conditions de stockage, plus particulièrement lors du transport, qui facilitent le développement bactérien et accentuent ainsi la contamination globale.

$\mathrm{Au}$ vu des résultats de la présente étude, il apparaît nécessaire d'agir prioritairement au niveau des éleveurs pour améliorer la qualité. Toutefois, la filière est complexe et fonctionne comme un ensemble, il est donc indispensable que tous les niveaux soient impliqués dans une démarche globale par le travail concerté des différents acteurs. Pour cela, il est intéressant de les impliquer au cours de trois étapes majeures : (i) la sensibilisation pour permettre de comprendre l'intérêt de la gestion de l'hygiène et de connaître les « bonnes pratiques » à mettre en œuvre; (ii) la mise en place de systèmes de contrôle pouvant jouer à la fois un rôle de surveillance et de conseil pour progresser dans la démarche ; (iii) la rémunération à la qualité servant d'encouragement et de système de reconnaissance envers les acteurs qui se sont impliqués.

De plus, les conditions de préservation et de transport du lait doivent être améliorées de manière à éviter la dégradation de la qualité initiale. Deux approches peuvent être envisagées : (i) la gestion des risques par le développement de la chaîne du froid ou, en tant que transition, l'utilisation du SLP ; (ii) la stérilisation finale du produit avant la vente par la mise en place de minilaiteries. Quelles que soient les solutions envisagées par les autorités ougandaises, elles devront combiner les deux éléments que sont l'amélioration de la qualité initiale et son maintien au cours du transport jusqu'au consommateur.

\section{Remerciements}

L'étude a été menée avec le soutien et la participation de l'ambassade de France en Ouganda dans le cadre du projet Fonds de solidarité prioritaires. A ce titre, les auteurs remercient M. Bellinguez, chef du projet Consultation agricole et structuration des filières (Casf), ainsi que Mme Baherle, responsable du service de Coopération et d'Action culturelle de l'ambassade de France en Ouganda en 2004. Les auteurs adressent également leurs remerciements à leurs partenaires ougandais de la DDA, en particulier à Mme A. Baguma, de l'Unbs, des universités de Mbarara et de Makerere. Enfin, ils remercient l'équipe du projet Casf pour son travail au sein de l'étude ainsi que l'ensemble des producteurs et des acteurs de la filière informelle pour leur disponibilité et les informations qu'ils leur ont communiquées. 


\section{BIBLIOGRAPHIE}

1. ALIGUMA L., NYORO J.K., 2004. Regoverning markets. Scoping study on dairy products, fresh fruits and vegetables. Kampala, Uganda, Ministry of Agriculture, $40 \mathrm{p}$.

2. ASPERGER H., 1993. Methods for recontamination control. Bull. int Dairy Fed., 281: 16-19.

3. ASPERGER H., 1993. Microbiology of pasteurized milk. Bull. int. Dairy Fed., 281: 14-16.

4. BELLINGUEZ A., 1994. Etude de l'impact des projets sur les systèmes de production laitiers périurbains à Bamako, Mali. Mémoire Ingénieur, Cnearc/Esat, Montpellier, France, p. 69-70.

5. BENNETT A, 2000. The lactoperoxidase system (LP-s) of milk preservation In: FAO E-mail Conf. Small-scale milk collection and processing in developing countries, 29 May- 28 Jul. 2000. FAO, Rome, Italy, p. 68-70.

6. CLAESSON O., 1994. The use of the lactoperoxidase system. In: Proc Regional workshop on raw milk handling and preservation in the Near East region, University of Alexandria, Egypt, 13-15 Sept. 1994. Rome, Italy, FAO, p. 26-40.

7. COUNCIL OF THE EUROPEAN COMMUNITY, 1992. Counci directive 92/46/EEC of 16 June 1992 laying down the health rules for the production and placing on the market of raw milk, heat-treated milk and milk-based products. European Community, www.europa.eu.int/com/ food/fs (30/03/05).

8. DABUSTI N., VANCAUTEREN D., 1999. Les systèmes d'élevage du district de Mbarara (Ouganda) et leur contribution à la filière laitière. Diagnostic, dynamiques d'évolution et recommandations pour l'action. Mémoire Ingénieur Cnearc/Esat, Montpellier, France, 252 p.

9. DE GRAAF T., ROMERO ZUNIGA J.J., CABALLERO M., DWINGER R.H., 1997. Microbiological quality aspects of cow's milk at a smallholder cooperative in Turrialba, Costa Rica. Revue Elev. Méd. vét Pays trop., 50 : 57-64.

10. DESVAUX S., 2001. Contraintes hygiéniques et sanitaires de la filière lait dans le district de Mbarara en Ouganda. Etude et propositions d'actions pour la maîtrise de la qualité du lait. Thèse Doct. vét., université de Nantes/ENV, France, 109 p.

11. EAST AFRICAN COMMUNITY, 1999. East African standards Unprocessed (raw) whole milk - Specification. Arusha, Tanzania, EAC, $14 \mathrm{p}$

12. FABOZZI L., VANCAUTEREN D., 2004. Milk producers associations in Mbarara district. Inventory and priority needs appraisal. Final report. Kampala, Ouganda, ACSS, 78 p.

13. FAO/OMS, 2000. Codex Alimentarius. Lait et produits laitiers, vol. 12, 2e édn. Rome, Italie, FAO, 129-136.

14. FAO/WHO, 2005. Reports of the ad hoc FAO/WHO exper consultations on risk assessment of microbiological hazards in food and related matters. Prepared by FAO and WHO, 14-19 March 2005. Buenos Aires, Argentina, CCFH, 9 p.

15. FAYE B., ALARY V., 2001. Les enjeux des productions animales dans les pays du Sud. Prod. Anim., $14: 3-13$.

16. FAYE B., CASTEL V., LESNOFF M., RUTABINDA D., DHALWA J., 2005. Tuberculosis and brucellosis prevalence survey on dairy cattle in Mbarara milk basin (Uganda). Prev. vet. Med., 67: 267-281.
17. FAYE B., LOISEAU G., 2002. Sources de contamination dans les filières laitières et exemple de démarches qualité. In : Actes atelier int. Gestion de la sécurité des aliments dans les pays en développement. Sources de contamination dans les filières laitières et exemples de démarches qualité, Montpellier, France, 11-13 déc. 2000. Montpellier, France, Cirad, cédérom.

18. GRIMAUD P., FAYE B., MUGARURA L., MUHOOZI E., BELLINGUEZ A., 2004. Identification of research activities for the dairy sector development in Uganda: systemic and participatory approaches. Uganda J. Agric. Sci., 9: 879-884.

19. GUIRAUD J.P., 1998. Analyse du lait. In : Micro-biologie alimentaire. Paris, France, Dunod, p. 387-413.

20. ISHARAZA W.K., 2002. Approaches to quality control of milk and dairy products in Uganda. In : Atelier sur la qualité des produits animaux dans les pays tropicaux, Rennes, France, 11-22 sept. 2001. Montpellier, France, Cirad-emvt, p. 83-85.

21. JOUZIER X., COHEN-MAUREL E., 1986. Manuel de référence pour la qualité du lait. Paris, France, Cidil, 199 p.

22. LANDO'LAKES, 2001. Report on the milk production market chain study. Kampala, Ouganda, Friend's Consult, 44 p.

23. LHOSTE F., RAMET J.P., 2004. Le lait de brousse. L'organisation de la collecte du lait dans les zones sans infrastructure laitière en Afrique de I'Ouest. Rome, Italie, FAO, 31 p.

24. LOISEAU G., 2002. Les tests de qualité du lait. In : Memento de I'agronome. Montpellier, France, Cirad, cédérom.

25. MAE, 2005. Afrique centrale et orientale. Paris, France, ministère des Affaires étrangères, www.diplomatie.gouv.fr/actu/actu.asp?DOS=12464 (26/02/05).

26. MEYER C., DENIS J.P., 1999. Elevage de la vache laitière en zone tropicale. Montpellier, France, Cirad, 313 p.

27. MONTEL M.C., BEUVIER E., HAUWUY A., 2003. Pratiques d'élevage, microflore du lait et qualités des produits laitiers. Prod. Anim., 16 : 279-282.

28. PISSANG TCHANGAI D., 2001. Evaluation de la qualité du lait et des produits laitiers dans les systèmes traditionnels de transformation au Tchad. In : Actes atelier int. Marchés urbains et développement laitier en Afrique subsaharienne, Montpellier, France, 9-10 sept. 1998. Montpellier, France, Cirad, p. 125-133.

29. ROZIER J., CARLIER V., BOLNOT F., 1984. Bases microbiologiques de l'hygiène. Paris, France, Sapaic, 232 p.

30. SAMARAJEEWA U., 1999. Manual on microbiological analysis. Kampala, Ouganda, UNBS, $125 \mathrm{p}$.

31. SOUKEHAL A.H., 1996. Amélioration de la collecte du lait par I'utilisation de la technique de réactivation du système lactoperoxydase. In : $2^{\mathrm{e}}$ journée scientifique et technique en agro-sylvo-pastoralisme, N’Djamena, Tchad, 9-12 déc. 1996. Rome, Italie, FAO, 4 p.

32. TOURETTE I., MESSAD S., FAYE B., 2002. Impact des pratiques de traite des éleveurs sur la qualité sanitaire du lait de chamelle en Mauritanie. Revue Elev. Méd. vét. Pays trop., 55 : 229-233.

33. WEBER, 1985. Réfrigération du lait à la ferme et organisation des transports. Rome, Italie, FAO, 216 p. (Production et santé animales $n^{\circ} 47$ )

Reçu le 11.10.2005, accepté le 29.05.2006 


\section{Summary}

Grillet N., Grimaud P., Loiseau G., Wesuta M., Faye B. Sanitary Quality of Raw Milk within the Commodity Subsector in Mbarara District and Kampala City in Uganda

The sanitary quality of raw milk is an important issue in Uganda for social, economical and health reasons. The present study carried out on the informal raw milk subsector of Uganda highlighted two main issues: (i) poor hygiene conditions from the production location all the way to the consumer; (ii) lack of an efficient preservation system to limit bacteria development during transportation to Kampala. The bacteria population reached very high levels close to $2 \times 10^{6}$ colony forming units per milliliter on the farm milk of Mbarara District in the southwestern region of the country, and these levels increased 150-fold during transportation to Kampala. The sector also includes rudimentary pasteurization units, where the overheated milk comes out bacteria-free. However, conservation over several days of the overheated milk makes this process potentially more dangerous than beneficial. Thus, the need for all the players of the sector to implement a strategy to improve milk quality can be two ways: (i) by changing common practices to ensure better hygiene conditions; (ii) by improving milk preserving through new methods such as cooling, small-scale pasteurization, or the use of the lactoperoxidase system. This study can help develop a technical and scientific basis to generate quality improvement actions in Uganda. But, whatever the strategy adopted by decision makers, it can only be implemented if all the stakeholders of the sector are involved.

Keywords: Milk - Quality - Food hygiene - Biological contamination - Preservation - Uganda.

\section{Resumen}

Grillet N., Grimaud P., Loiseau G., Wesuta M., Faye B. Calidad sanitaria de la leche cruda a lo largo de la filial en el distrito de Mbarara y de la ciudad de Kampala (Uganda)

La calidad sanitaria de la leche cruda es una problemática importante en Uganda, tanto por razones socio-económicas como sanitarias. El estudio llevado a cabo sobre la filial informal de la leche cruda en Uganda mostró dos puntos críticos principales para la calidad de la leche: la falta de higiene entre el lugar de producción y el lugar de consumo y la ausencia de un sistema eficaz de conservación que limite el desarrollo de las bacterias durante el transporte hacia Kampala. En efecto, la carga microbiana alcanzó niveles muy elevados, cerca de $2 \times 10^{6}$ unidades formando colonias por mililitro de leche de finca en la meseta lechera de Mbarara al sudoeste del país, que el transporte hacia la capital Kampala multiplicó por un factor de alrededor 150. En el seno de esta filial informal, existen unidades artesanales Ilamadas de pasteurización. La leche que éstas producen esta ciertamente libre de bacterias al final de la producción, pero la conservación durante varios días de este producto sobrecalentado podría transformar éste en un proceso más peligroso que beneficioso. Estas constataciones deben alertar a los actores de la filial informal sobre la necesidad de poner en marcha una estrategia para mejorar la calidad de la leche, mediante una modificación de las prácticas corrientes, para que respeten la higiene y por una mejoría de la conservación de la leche mediante tecnologías, tales como la refrigeración, la pasteurización a pequeña escala o entonces el uso de un sistema de lactoperoxidasa. Este estudio permitió la adquisición de bases técnicas y científicas para apoyar las acciones orientadas a mejorar la calidad de la leche cruda en Uganda. Cualquiera que sea la estrategia adoptada por las autoridades, ésta solo podrá ser puesta en marcha si ella implica al conjunto de los actores de la filial.

Palabras clave: Leche - Calidad - Higiena de los alimentos Contaminación biológica - Preservación - Uganda. 